Klein, W. (1998). Ein Blick zurück auf die Varietätengrammatik. In U. Ammon, K.J. Mattheier \& P.H. Nelde (Eds.), Sociolinguistica. Internationales Jahrbuch für Europäische Soziolinguistik, 12, 22-38. Tübingen: Niemeyer.

Wolfgang Klein

\title{
Ein Blick zurück auf die Varietätengrammatik
}

Plus ça change, plus c'est la même

chose.

\section{Weshalb ein Blick zurück?}

Wenn man, das nahe Ende des Säkulums vor Augen, den Blick auf die Geschichte des eigenen Faches richtet, so mag es dafür, wie bei jeglicher Beschäftigung mit dem Vergangenen, drei Beweggründe geben. Der erste und elementarste ist der Wunsch zu erfahren oder sich zu vergegenwärtigen, wie es denn eigentlich gewesen ist - der Wunsch, nicht nur zu wissen, was der Fall ist, sondern auch, was der Fall war. Der zweite Beweggrund ist die Vorstellung, man könne das, was ist, aus dem erklären, was war. Der dritte schließlich ist der noble Gedanke, man vermöchte aus dem Studium des Vergangenen vielleicht doch auch etwas für das eigene Handeln lernen.

Unter diesen Beweggründen ist der erste der selbstverständlichste - es ist die Wissbegierde, ohne die es keine Wissenschaft gäbe. Der zweite ist weniger selbstverständlich, aber gleichwohl verbreitet und vielen fraglos. Für Hermann Paul, in dessen erstmals 1880 erschienenen "Principien der Sprachgeschichte" die Sprachtheorie des vergangenen Jahrhunderts gipfelt, war es selbstverständlich, dass es nur eine wirklich wissenschaftliche Betrachtung der Sprache geben könnte, nämlich die geschichtliche; eine Sprachforschung, die sich nur mit dem Gegenwärtigen befasst, mag wohl die Fakten deskriptiv gut erfassen; aber sie kann nicht ihren Zusammenhang erklären. Eine synchrone Betrachtung mag sagen, wie es ist, aber nicht, warum es so ist; deshalb ist sie nicht wirklich wissenschaftlich:

Es ist eingewendet, daß es noch eine andere wissenschaftliche Betrachtung der Sprache gäbe, als die geschichtliche. Ich muss das in Abrede stellen. Was man für eine nichtgeschichtliche und doch wissenschaftliche Betrachtung der Sprache erklärt, ist im Grunde nichts als eine unvollkommen geschichtliche, unvollkommen teils durch Schuld des Betrachters, teils durch Schuld des Beobachtungsmaterials. Sobald man über das blosse Konstatieren von Einzelheiten hinausgeht, sobald man versucht, den Zusammenhang zu erfassen, die Erscheinungen zu begreifen, so betritt man auch den geschichtlichen Boden, wenn auch vielleicht ohnee sich klar darüber zu sein. (Paul 1886: 19f.). 
Heute, mehr denn als ein Jahrhundert später, ist uns dieser Gedanke etwas fremd geworden, aber auch wiederum nicht ganz: der Zusammenhang zwischen den beobachteten Einzelfakten ist uns durch allgemeine Stukturprinzipien bestimmt, und was sich diesen allgemeinen Prinzipien entzieht, sind, so nimmt man zumindest gerne an, nicht zum geringsten Teil Residuen einer unvollkommenen historischen Entwicklung. Das deutsche Perfekt zum Beispiel hat weitgehend seine alte Funktion verloren; es hat in vielen Varietäten der Gegenwartssprache die Aufgabe des alten Präteritums übernommen und dient dazu, Ereignisse in der Vergangenheit darzustellen. Aber diese Entwicklung ist nicht abgeschlossen, es gibt einige Verwendungen, beispielsweise in der Interaktion mit Adverbien, in denen die alte Bedeutung durchscheint: das neue System hat einige Störstellen. Die Sprachgeschichte erklärt nicht die Regel, aber sie erklärt die Ausnahmen, und so hat denn die geschichtliche Betrachtung immer noch einen gewissen Erklärungswert. Der dritte Beweggrund sich mit Vergangenem zu befassen, die Vorstellung nämlich, man könne aus der Geschichte lernen, wie man es besser macht, spielt im Falle der Sprachgeschichte nur eine geringe Rolle. Zwar muss jeder Unvoreingenommene zugeben, dass unsere Altvorderen uns in vielerlei Hinsicht eine Menge an sprachlichem Müll hinterlassen haben - ich nenne nur die Genusunterscheidung, die Kasusparadigmen oder die unterschiedliche Verbstellung in Hauptund Nebensatz, von der Orthographie ganz zu schweigen. Aber die Aussichten, dies in Hinkunft verbessern zu können, sind gering. Vielleicht kann uns die Sprachgeschichte etwas lehren; aber bestimmt nicht, es besser zu machen.

Anders ist dies freilich - vielleicht -, wenn man sich nicht der Geschichte der Sprache selbst zuwendet, sondern den Bemühungen, die Sprache wissenschaftlich zu erforschen. Auch hier gelten die drei nämlichen Beweggründe: man mag wissen oder, falls man selbst dabei war, sich vergegenwärtigen wollen, wie es gewesen ist; man mag verstehen wollen, weshalb sich das Fach dahin entwickelt hat, wo es jetzt steht, und ob es dahinter womöglich irgendeine Logik gibt. Und man mag die Hoffnung hegen, aus den Fehlern der Vergangenheit zu lernen. In diesem Essay will ich einen kleinen Teil der Entwicklung unseres Faches betrachten, an dem ich selbst beteiligt war: das Fatum der "Varietätengrammatik", eines Instrumentes, das darauf abzweckt, die sprachliche Variabilität systematischer und präziser Analyse zugänglich zu machen. Dieser Versuch, so kann man heute sagen, war nicht sehr erfolgreich. Ich sehe dies mit einem gewissen Bedauern, das sich aber, da ich selbst seit langem auf anderen Gebieten arbeite, in Grenzen hält. Ob sich aus diesem Blick in die Vergangenheit etwas lernen läßt, das mag ein jeder für sich sehen.

Die Varietätengrammatik war ein Versuch, zwei völlig disparate Entwicklungen der Linguistik zu Beginn der Siebzigerjahre miteinander zu verbinden - nämlich Ergebnisse und konzeptuelle Überlegungen der Soziolinguistik einerseits und der Theorie der formalen Grammatik anderseits. Erstere sind vor allem durch die Untersuchungen von William Labov (1972a, b) repräsentiert, letztere durch die Arbeiten generative Grammatik, wie sie von Chomsky und anderen entwickelt worden war. Disparat waren diese Entwicklungen in zumindest dreierlei Hinsicht:

\section{A. Im empirischen Vorgehen}

In der formalen Grammatik, sei es in der generativen Syntax oder in der formalen Semantik, stützt sich der Linguist im wesentlichen auf seine (gelegentlich durch Befragung einiger Freunde angereicherte) Intuitionen - in den selbstironischen Worten eines der bedeutensten Linguisten jener Tage: "Write a grammar of what you find in your heart!" (J. R. Ross). Der 
Soziolinguist hingegen, wie jeder, der die sprachliche Variation erforschen will, sammelt mit Fragebogen oder Tonband möglichst reiche Belege aus der Produktion oder, freilich viel seltener, dem Verstehen von Sprechern unterschiedlicher Herkunft, analysiert diese und versucht, irgendwelche Regelmäßigkeiten darin zu entdecken. Die Befragung der eigenen Intuition verwirft er oder läßt sie nur als vorläufige Technik von einem gewissen heuristischen Wert gelten.

\section{B. In der Darstellung der Analyseergebnisse}

In der formalen Grammatik bedient man sich, mit unterschiedlichem Geschick, bestimmter algebraischer Methoden, um die beobachtbaren Gesetzlichkeiten präzise und explizit darzustellen. Das bekannteste Beispiel sind sicherlich die verschiedenen generativen Grammatiken, die damals im Schwange waren - kontextfreie Grammatiken, Kategorialgrammatiken, Transformationsgrammatiken, um nur einige zu erwähnen. In der Soziolinguistik werden die Resultate dagegen meist informell dargestellt, in schlichter, mehr oder minder klarer Prosa, die gelegentlich durch Tabellen, Diagramme und Statistiken angereichert ist.

\section{In unterschiedlichen Vorstellungen vom Gegenstand der sprachwissenschaft-lichen Forschung}

Ziel der Bemühungen des Linguisten ist es, nach einem bekannten Wort Chomskys, das sprachliche Wissen des idealen Sprechers zu rekonstruieren. Die Variabilität, sei sie nun sozial oder sonstwie bedingt, spielt keine oder jedenfalls keine sonderlich wichtige Rolle; sie ist eigentlich eher ein Ärgernis, ein Störfaktor, von dem man zweckmäßiger Weise abstrahiert. Gegenstand der soziolinguistischen Forschung hingegen sind gerade die Unterschiede im sprachlichen Wissen und, damit zusammenhängend, im sprachlichen Verhalten der einzelnen, die eine Sprache sprechen.

Diese drei Faktoren stehen in einem engen Zusammenhang. Wenn man das Wissen des Sprechers, insoweit es sich nicht von dem anderer Sprecher unterscheidet, rekonstruieren will, so ist es nicht erforderlich, breitgestreute Erhebungen in verschiedenen Kreisen der Bevölkerung zu machen. Vielmehr muss man versuchen, so einfach wie möglich Zugang zu diesem Wissen zu finden, und da ist die Befragung der eigenen Intuitionen (Ist "wüscht' ein deutsches Wort? Ist 'Ein Buch gekauft haben soll er keins'? grammatisch wohlgeformt? Sind die Sätze 'Englisch spricht man in Australien' und 'In Australien spricht man Englisch' gleichbedeutend?) zumindest ein ökonomischer Weg. Wenn man "die deutsche Sprache der Gegenwart" in all ihren vielen dialektalen, sozialen, stilistischen und sonstigen Spielarten wissenschaftlich beschreiben will, dann braucht man repräsentative Daten aus all diesen Varietäten, und man kann "die deutsche Sprache" als soziales System nicht einfach durch eine formale Grammatik üblichen Zuschnitts darstellen, sondern bestenfalls durch ein Gefüge solcher Grammatiken.

Aber dieser Zusammenhang ist, jedenfalls was die beiden ersten beiden Punkte betrifft, kein notwendiger. Intuitionen im gedachten Sinn geben keinen direkten, unverstellten Zugang zum sprachlichen Wissen. Es sind - zumeist sehr schlecht kontrollierte - metasprachliche Urteile und damit eher untypische Manifestationen der menschlichen Sprachfähigkeit. Normalerweise spricht und versteht man: man gibt kein Urteil darüber ab, ob bestimmte Äußerungen grammatisch oder synonym sind. Auch wenn man an dem Ziel festhält, es komme darauf an, die Kompetenz des idealen Sprechers zu rekonstruieren, ist es sinnvoll, ja 
erforderlich, andere, verläßlichere Daten hinzuzuziehen. Umgekehrt gibt es keinen wirklich zwingenden Grund, sich bei der Beschreibung einer "Sprache als System von Varietäten" mit informellen, nicht sehr expliziten Charakterisierungen zu bescheiden; es sollte möglich sein, mehr oder minder verschiedene Grammatiken zu einem präzisen System zu verbinden, das Gemeinsamkeiten und Verschiedenheiten der einzelnen Varietäten genau abbildet. Ebendies zu leisten war das Ziel der Varietätengrammatik. Wie dies geschieht, wird in Abschnitt 3 dargestellt. Zuvor will ich jedoch etwas näher auf die oben kurz erwähnten unterschiedlichen Vorstellungen von der Aufgabe der Sprachwissenschaft eingehen - Sprache als Wissen des idealen Sprechers und Sprache als komplexes soziales System. Dabei wird sich zeigen, dass sich das Problem der Variabilität und ihrer präzisen Beschreibung bei beiden Konzeptualisierungen letztlich nicht sehr unterschiedlich stellt.

\section{Was wollen wir eigentlich?}

Der vierten Auflage seiner "Prinzipien", die im Jahre 1909 erschien, schickte Hermann Paul ein weiteres Vorwort voraus, das folgendermaßen beginnt:

Von der neuen Auflage wird man vor allem eine Auseinandersetzung mit dem ersten Bande von Wundts Völkerpsychologie (Leipzig 1900, ${ }^{2} 1904$ ) erwarten. Leider kann ich mich diesem Werke gegenüber, so viele Anregungen es auch im einzelnen bringt, doch in den Hauptpunkten nur ablehnend verhalten.(..)

Der Gegensatz zwischen Wundt und mir beruht nicht so sehr darauf, daß ich mich an die Psychologie Herbarts angelehnt habe (...) während Wundt sein eigenes System zugrundelegt (...) eine viel tiefere und breiter Kluft trennt uns, die sich auf keine Weise überbrücken lässt, in Folge der beiderseitigen Stellung zur sogenannten Völkerpsychologie.

Wundt stellt, wie schon der Gesamttitel seines grossen Werkes zeigt, die Völkerpsychologie neben die Individualpsychologie. (...) Die Veränderungen der Sprache erfolgen nach ihm durch Veränderungen in der Volksseele, nicht durch solche in den Einzelseelen. Das Problem, welches für mich im Mittelpunkt der Untersuchung steht, die Frage, wie sich die Wechselwirkung der Individuen unter einander vollzieht, ist für Wundt überhaupt kein Problem.(...) Auf diese Weise kann meiner Überzeugung nach kein volles Verständnis der Sprachentwicklung gewonnen werden.

Die Rede von "Einzelseele" und "Volksseele" kommt uns heute etwas altfränkisch vor; wir haben uns inzwischen an so präzise und wohldefinierte Begriffe wie "I-language" und "Elanguage" (Chomsky 1986), "individual competence - social competence", "modularity of mind" und dergleichen gewöhnt. Was Paul meint, wird jedoch recht deutlich aus dem ersten Paragraphen von Wundts Werk, mit dem er sich nicht auseinandersetzen möchte:

Die Psychologie in der gewöhnlichen und allgemeinen Bedeutung dieses Wortes sucht die Tatsachen der unmittelbaren Erfahrung, wie sie das subjektive Bewußtsein uns darbietet, in ihrer Entstehung und in ihrem wechselseitigen Zusammenhang zu erforschen. In diesem Sinn ist sie INDIVIDUALPSYCHOLOGIE. Sie verzichtet durchgängig auf eine Analyse jener Erscheinungen, die aus der geistigen Wechselwirkung einer Vielheit von Einzelnen hervorgehen. Eben deshalb aber bedarf 
sie einer ergänzenden Betrachtung, die wir der VÖLKERPSYCHOLOGIE zuweisen. Demnach besteht die Aufgabe dieses Teilgebiets der Psychologie in der Untersuchung DERJENIGEN PSYCHISCHEN VORGÄNGE, DIE DER ALLGEMEINEN ENTWICKLUNG MENSCHLICHER GEMEINSCHAFTEN UND DER ENTSTEHUNG GEMEINSAMER GEISTIGER ERZEUGNISSE VON ALLGEMEINGÜLTIGEM WERT ZUGRUNDELIEGEN. (Wundt 1904, 1).

Es gibt also so etwas wie den Einzelnen mit all seinen kognitiven und emotionalen Fähigkeiten - die Einzelseele -, und die Aufgabe der "Individualpsychologie" ist es, die Eigenschaften der Einzelseele mit den Methoden der experimentellen Psychologie, deren Hauptbegründer Wilhelm Wundt ja war, zu erforschen. Daneben aber gibt es seelische Erscheinungen, die über das hinausgehen, was im Kopf ("mind/brain") des Einzelnen abgelagert sind - jene Erscheinungen, die sich erst aus dem Zusammenwirken der Einzelseelen ergeben und deren Erforschung in den Bereich der "Völkerpsychologie" fällt. Zu diesen Erscheinungen zählen nach Wundt vor allem "Sprache, Mythos, Sitte". Sie bilden das Thema seiner monumentale, zehnbändigen "Völkerpsychologie", deren erste beiden Bände der Sprache gewidmet sind. Der religiöse oder wissenschaftliche Glaube einer bestimmten Gemeinschaft, etwa der Christen oder jener der Linguisten, ist mehr als das, was der Einzelne, der zu dieser Gemeinschaft zählt, davon weiss: er ist eine genuin soziale Gegebenheit. Folglich lässt er sich auch nicht befriedigend mit den Methoden der experimentellen Psychologie studieren. Diese Methoden lassen sich sinnvoll nur auf das anwenden, was Wundt "elementare Bewußtseinsprozesse" nennt. Dies schließt nicht aus, dass sich auch bestimmte linguistische Fakten auf diese Weise studieren lassen - beispielsweise die Segmentation des Schallstroms oder artikulatorische Prozesse, überhaupt die gesamte Phonetik. Aber die Art und Weise, wie in einer bestimmten Sprache Temporalität oder Possessivität ausgedrückt werden, wie ihre Argumentstruktur ist und welche Paradigmen der Nominalflektion vorkommen - all dies ist im Prinzip ganz unabhängig davon, wie es im Kopf eines beliebigen Sprechers gespeichert und wie dieser sein Wissen in einer gegebenen Situation aktiviert. Die Sprache, so wie Wundt sie auffasst, hat nicht bloss eine soziale Seite das würde ein jeder zugeben -, sondern sie ist sozial konstituiert, sie ist GENUIN SOZIAL. Dies hat erhebliche Konsequenzen für ihre Erforschung: der Gegenstand der Sprachwissenschaft ist ein soziales Phänomen, das man nicht angemessen erfassen kann, wenn man lediglich seine Widerspiegelung im Individuum untersucht. Eines ist die Sache selbst, ein anderes das, was irgendjemand davon weiss.

Hermann Paul sieht dies ganz anders. Zwar betrachtet er die Sprache gleichfalls als eine psychische Gegebenheit, und die Sprachwissenschaft als einen Teil der Psychologie - aber der Individualpsychologie:

(Es ist eine) TATSACHE VON FUNDAMENTALER BEDEUTUNG, DIE WIR NIEMALS AUS DEM AUGE VERLIEREN DÜRFEN, DAß ALLE REIN PSYCHISCHE WECHSELWIRKUNG SICH NUR INNERHALB DER EINZELSEELE VOLLZIEHT. ALLER VERKEHR DER SEELEN UNTEREINANDER IST NUR EIN INDIREKTER, AUF PHYSISCHEM WEGE VERMITTELTER. ES bleibt also dabei, es kann nur eine individuelle Psychologie geben, der man keine Völkerpsychologie oder wie man es sonst nennen mag gegenüber stellen darf. (Paul 1909, S. 12s)

Alles Psychische, Konzeptuelle, Mentale ist nur im Individuum. Zwischen den Individuen mag es Wechselwirkungen geben; aber diese sind rein physischer Natur, irgendwie 
modulierte Schallwellen oder Krakel auf dem Papier, die man mit Ohren oder Augen wahrnehmen kann; sie sind objektiv vorhanden, messbar und beschreibbar, aber eigentlich für die Erforschung psychischer Erscheinungen, und damit auch der Sprache, nicht von besonderem Interesse. Der Gegenstand der Sprachwissenschaft ist das, was der Sprecher im Kopf hat.

Die Sprachtheorie des vergangenen Jahrhunderts kulminiert also in zwei radikal entgegengesetzten Konzeptualisierungen dessen, was man unter dem Gegenstand sprachwissenschaftlicher Forschung verstehen soll: der "Wundt-Sprache", derzufolge die Sprache eine soziale Hervorbringung ist, die das Wissen des Einzelnen transzendiert, und der "PaulSprache", derzufolge alles an der Sprache Wesentliche im Kopf des Individuums zuhause ist. Dies war das Bild vor vor fast hundert Jahren. Schaut man zurück auf das, was seither gekommen ist, so haben wir, was die Objektkonstitution unser Disziplin betrifft, anscheinend nicht viel dazugelernt. Mit der hier gebührenden Vereinfachung kann man sagen, dass für die Geschichte unseres Faches in diesem Jahrhundert zwei Betrachtungsweisen bestimmend waren. Die erste ist die der strukturalistischen Tradition, deren Anfänge gewöhnlich auf Ferdinand de Saussure zurïckgeführt wird. Danach ist der zentrale Gegenstand der Linguistik ein abstraktes soziales System, ein "fait social", die LANGUE, die von den einzelnen Sprechern mehr oder minder geteilt wird, obwohl keiner sie vollständig besitzt. Was individuell ist, ist zum ersten die FACULTÉ DE LANGAGE, d.h. die allen Menschen von Natur aus gegebene Fähigkeit, sich eine "langue" oder auch deren mehrere in gewissen Grenzen anzueignen, und zum zweiten der Gebrauch, den man von der so erworbenen Fertigkeit in bestimmten kommunikativen Situationen macht (die PAROLE). Diese Denkweise bestimmte einen großen Teil der Linguistik in der ersten Hälfte dieses Jahrhunderts (soweit sich die Sprachwissenschaftler überhaupt den Kopf über das zerbrechen, was sie eigentlich tun), und sie wird nach wie vor viele - explizit oder doch stillschweigend - von vielen vertreten.

Der zweite Traditionsstrang wird gewöhnlich auf Noam Chomsky und seine Arbeiten aus den Fünfzigerjahren zurückgeführt. Man pflegt in diesem Zusammenhang immer die folgende Stelle zu zitieren, und das will ich auch hier nicht verfehlen:

Linguistic theory is concerned primarily with an ideal speaker-listener, in a completely homogeneous speech-community, who knows its language perfectly and is unaffected by such grammatically irrelevant conditions as memory limitations, distractions, shifts of attention and interest, and errors (randomly or characteristic) in applying his knowledge of the language in actual performance. (Chomsky 1965, S. 3).

Chomksy meint sehr wohl, dass es so etwas wie "die Sprache einer sozialen Gemeinschaft" gibt ("its language"), die der einzelne im Idealfall perfekt, in der Realität mehr oder minder gut kennt. Nur ist diese nicht der Gegenstand der linguistischen Theorie: das ist vielmehr ihr Reflex im Kopf des Einzelnen. Die "Sprache einer Gemeinschaft" hingegen, die "E-Sprache" späterer Arbeiten (Chomsky 1985), ist ein ziemlich schlecht definiertes Objekt, das der wissenschaft-lichen Erforschung nicht recht zugänglich ist - im Gegensatz zur "I-Sprache", der im Kopf gespeicherten Grammatik des idealisierten Individuums. Dies kommt der Vorstellung Hermann Pauls sehr nahe, der auch nicht bestritten hätte, dass die deutsche Sprache mehr umfasst als das, was irgendjemand zu einer gegebenen Zeit davon weiss; schließlich hat Paul sich genug mit historischen und dialektalen Varietäten des Deutschen beschäftigt. Aber als soziale Gegebenheit, als "Wundt-Sprache", ist sie kein primärer Gegenstand der Wissenschaft.

In der Tat, was ist schon "die deutsche Sprache" als soziales Objektivgebilde? Sie ist sicher 
kein "système ou tout se tient", in Saussures berühmter Formulierung, sondern ein Amalgam höchst verschiedener lexikalischer Einheiten und Regularitäten, gegliedert nach Dialekten, Soziolekten, Registern und sonstigen Faktoren der Variation. Die "Wundt-Sprache" ist nichts Einheitliches, sie stellt sich als ein komplexes System von Varietäten dar, die viele Gemeinsamkeiten, aber auch viele Verschiedenheiten aufweisen und die gleitend in andere, benachbarte "natürliche Sprachen" übergehen. Wie kann man ein solches System von Systemen präzise beschreiben? Sicher nicht durch eine einheitliche Grammatik; vielmehr benötigt man parallel zu den Varietäten ein System von Grammatiken, deren Zusammenhang untereinander klar definiert ist.

Lät sich die Aufgabe, ein solches System von aufeinander bezogenen Grammatiken zu entwickeln, lösen, indem man die "Paul-Sprache" zum zentralen Gegenstand linguistischer Bemühungen erklärt? Nein, denn dies hieße, nur das untersuchen zu wollen, was der Einzelne von der "Wundt-Sprache" weiss. Dies reduziert das Problem, aber nur im Grad, nicht im Grundsatz, und dies um den Preis, dass viele offenkundige sprachliche Fakten und Regularitäten aus dem Blick geraten. Es reduziert das Problem, weil das Wissen des Einzelnen stets nur einen kleinen Ausschnitt aus der "Wundt-Sprache" umfasst; man spricht und versteht vielleicht einige Dialekte und Register, und dies unterschiedlich gut. Darunter ist oft auch jene Varietät, die in normativen Grammatik als Standard vorgegeben wird, oder jedenfalls wesentliche Ausschnitte davon. Aber diese Varietät ist natürlich nur sozial vor den anderen ausgezeichnet, sie ist nicht "das Deutsche" oder "das Englische". Man kann sich nun einen "idealen Sprecher" als eine Person vorstellen, die alle Varietäten des Deutschen überblickt; aber dann verschwindet der Unterschied zur "Wundt-Sprache". Oder man nimmt jenen für den idealen Sprecher, dessen Kompetenz sich in einer Varietät erschöpft. Aber das ist gleichbedeutend damit zu sagen, dass man einfach nur eine Varietät, einen Dialekt etwa, untersucht - einen mehr oder minder gut motivierten Ausschnitt aus der Kompetenz eines normalen Sprechers.

Die Lösung läge darin, eine Art der grammatischen Beschreibung zu entwickeln, bei der die Abbildung einer einzigen, in sich homogenen Varietät als Grenzfall erscheint, die sich aber ohne Verlust an Präzision und Explizitheit auf Systeme verwandter Varietäten anwenden läßt. Ein solches Verfahren ist die Varietätengrammatik.

\section{Was ist die Varietätengrammatik?}

Sprachliche Varietäten unterscheiden sich in den unterschiedlichsten Eigenschaften - in der Phonologie, in der Syntax, in den lexikalischen Einheiten, in verschiedenen pragmatischen Aspekten. Im vorliegenden Zusammenhang geht es nur um grammatische Eigenschaften, und auch hier will ich, dies freilich nur zum Zweck der Illustration, lediglich einige einfache Beispiele aus der Syntax betrachten. Im Prinzip läßt sich dasselbe Verfahren auf alle möglichen grammatischen Erscheinungen anwenden, sofern sie überhaupt einer Beschreibung durch formale Regeln zugänglich sind. Die folgende Darstellung ist ganz informell, und sie soll, ohne präzise Definitionen, lediglich eine anschauliche Vorstellung davon vermitteln, was eine Varietätengrammatik ist. (Eingehende Beschreibungen finden sich beispielsweise in Habel 1979 und Klein 1974, 1988; Habel 1979 enthält auch einen kritischen Vergleich zu anderen formalen Methoden der Varietätenanalyse.)

\subsection{Die Grundidee}


Betrachten wir als Beispiel die Grundwortstellung im Satz - Verb und ein, zwei oder drei Argumente. Nehmen wir an, in einer Varietät $\mathrm{V}_{1}$ steht das Verb am Ende (V-End), in einer zweiten Varietät $V_{2}$ hingegen nach dem ersten Argument (V-Zweit). Dabei ist vollkommen gleichgültig, ob diese beiden Varietäten zur Kompetenz eines Individuums zählten, also beispielsweise zwei Soziolekte sind, die dieser Sprecher gleichermaßen beherrscht und zwischen denen er nach Gegebenheiten der Situation wechseln kann, oder ob es beispielsweise zwei mehr oder minder weit auseinanderliegende historische Entwicklungsstufen derselben Sprache darstellen, die man aus irgendeinem Grunde vergleichen möchte. In jedem Fall ergeben sich rein deskriptiv für den einfachen Satz die folgenden Muster:

(1) Zwei Grundwortstellungen

$$
\mathrm{V}_{1}
$$

Argument

Argument

Argument

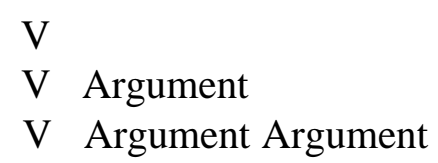

$\mathrm{V}_{2}$
Argument
Argument
V
Argument Argument Argument V

Für jede dieser beiden Varietäten kann man nun eine eigene formale Grammatik schreiben, die unter anderem die jeweiligen Satzmuster abzuleiten erlaubt. Dafür gibt es verschiedene Möglichkeiten. Die einfachste davon sind gewöhnliche Konstituentenstrukturgrammatiken, die man durch (kontextfreie) Phrasenstrukturregeln beschreiben kann (sogenannte Typ-2Grammatiken). Im Beispiel könnte dies so aussehen:

$$
\mathrm{G}\left(\mathrm{V}_{1}\right)
$$

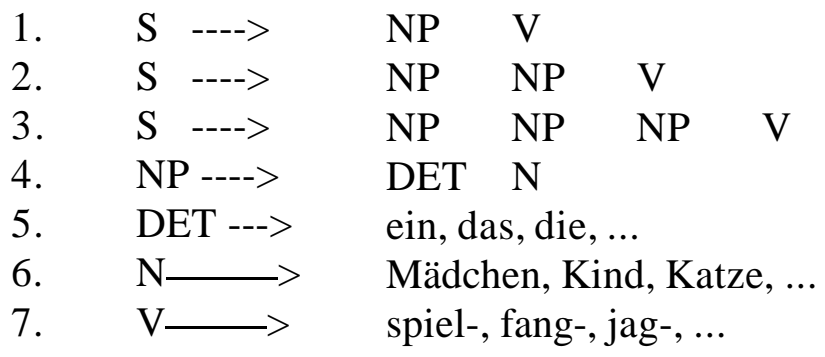

(3) $\mathrm{G}\left(\mathrm{V}_{2}\right)$
1. $\quad \mathrm{S} \quad--->>$
NP V
2. $\quad \mathrm{S} \quad--->>$
NP $\quad \mathrm{V} \quad \mathrm{NP}$
3. $\quad \mathrm{S} \quad--->$
NP $\quad \mathrm{V} \quad \mathrm{NP} \quad \mathrm{NP}$
4. NP ---->
DET N
5. DET --->
ein, das, die, ...
6. $\mathrm{N} \longrightarrow$
Mädchen, Kind, Katze, ... 
7. $\quad \mathrm{V} \longrightarrow \quad$ spiel-, fang-, jag-,

Es ist leicht zu sehen, dass beide Minigrammatiken übergeneralisieren, d.h. neben zulässigen Strukturen wie die Katze jag- ein Kind auch unzulässige erzeugen, z.N. die Katze spiel- ein Mädchen; auch wird die Flexion weder beim Verb noch beim Nomen erfasst. Das soll uns aber hier nicht weiter stören. Beide Grammatiken unterscheiden sich nur in zwei Regeln, nämlich der jeweils zweiten und dritten. Dies reflektiert den Umstand, dass beide Varietäten eben auch sehr ähnlich sind. Wie kann man Ähnlichkeit und Verschiedenheit abbilden?

Das einfachste Verfahren besteht darin, beide Grammatiken zu einer einzigen (der "Bezugsgrammatik", die als tertium comparationis dient) zu vereinigen und die einzelnen Regeln nach ihrer Zugehörigkeit zu einer bestimmten Varietät zu markieren oder, wie Bierwisch 1976 vorschlägt, zu "konnotieren”. In diesem Fall sähe das Ergebnis so aus:

(4) Varietätengrammatik (kategorial) für $\mathrm{V}_{1}$ und $\mathrm{V}_{2}$

\begin{tabular}{|c|c|c|c|c|c|c|}
\hline 1. $S \quad--->$ & NP & $\mathrm{V}$ & & & + & + \\
\hline 2.S. $\longrightarrow$ & $\mathrm{NP}$ & $\mathrm{V}$ & NP & & + & - \\
\hline 3.S. $\longrightarrow$ & NP & NP & $\mathrm{V}$ & & - & + \\
\hline 4. $\mathrm{S} \longrightarrow$ & NP & $\mathrm{V}$ & $\mathrm{NP}$ & $\mathrm{NP}$ & + & - \\
\hline 5. S $\longrightarrow$ & NP & NP & NP & $\mathrm{V}$ & - & + \\
\hline 6. NP-----> & DET & $\mathrm{N}$ & & & + & + \\
\hline 7. DET ---> & \multirow{3}{*}{\multicolumn{4}{|c|}{$\begin{array}{l}\text { ein, das, die, } \\
\text { Mädchen, Kind, Katze, ... } \\
\text { spiel-, fang-, jag-, ... }\end{array}$}} & + & + \\
\hline 8. N $\quad--->$ & & & & & + & + \\
\hline 9. $\mathrm{V} \longrightarrow$ & & & & & + & + \\
\hline
\end{tabular}

Die erste Regel und die letzten vier Regel sind für beide Varietäten sind gleich, die Unterschiede zeigen sich nur in der "Bewertung" der Regeln 2 - 5. Das Beispiel ist natürlich extrem einfach und insofern sicher für jeden praktischen Zweck inadäquat. Es sollte aber deutlich sein, dass man sowohl die Zahl und Art der Regeln wie auch der Varietäten nach Belieben anreichern kann, um so den tatsächlichen empirischen Gegebenheiten besser Rechnung tragen zu können - immer im Modell der Phrasenstrukturgrammatik. Es gibt jedoch zwei klare Unzulänglichkeiten eines solchen Vorgehens. Zum ersten sind die Übergänge zwischen Varietäten oft nicht kate-gorial, sondern gleitend. Dies wird besonders deutlich, wenn es sich um Entwicklungsstufen handelt, bei denen sich ein grammatisches Muster allmählich in ein anderes entwickelt. Dies gilt sowohl für die Sprachgeschichte wie auch für den Spracherwerb, bei dem ein Einzelner durch verschiedene verwandte Entwicklungsstadien ("Lernervarietäten") läuft. Das zweite Problem ergibt sich aus der Adäquatheit der Regeln selbst: man hat im Beispiel nicht das Empfinden, dass der Unterschied Verb-Zweit gegenüber Verb-End wirklich angemessen abgebildet wird, weder in den einzelnen Varietäten selbst noch in der übergreifenden "kategorialen Varietätengrammatik". Das dritte Problem rührt aus der Abhängigkeit von Regelanwendungen. Diese drei Probleme und ihre Lösung sollen nun kurz diskutiert werden. 


\subsection{Drei Probleme}

\section{A. Gleitende Übergänge}

Dieses Problem lässt sich lösen, wenn man den einzelnen Regeln nicht einfach ein "ja" oder "nein" für jede Varietät zuordnet, sondern eine Zahl zwischen 0 und 1, die die Wahrscheinlichkeit der Anwendung dieser Regel ausdrückt. Der Wert 1 bedeutet, dass die Regel angewandt wird, wenn immer dies in einer Ableitung möglich ist: die Regel ist obligatorisch. Hingegen heißt 0, dass die Wahrscheinlichkeit ihrer Anwendung eben Null ist, d.h. dass sie nie angewandt wird. Mit anderen Worten, sie ist in der betreffenden Varietät nicht verwirklicht. Diese Werte drücken also gleichsam das "Gewicht" aus, das die jeweilige Regel im Vergleich zu anderen, die alternativ angewandt werden könnten, in einer bestimmten Varietät hat. Die Alternativen sind durch alle Regeln gegeben, die dasselbe Symbol expandieren. In unserem Beispiel ist dies S: sie bilden gemeinsam einen "Regelblock"; da eine von ihnen angewandt werden MUSS, ist die Gesamtwahrscheinlichkeit für den ganzen Block 1 - wie immer sich diese Gesamtwahrscheinlichkeit auf die einzelnen verteilen mag.

Nehmen wir beispielsweise an, $\mathrm{V}_{1} \ldots \mathrm{V}_{5}$ seien fünf Entwicklungsstufen einer Sprache, und die empirische Analyse habe die folgenden Wahrscheinlichkeiten (wie üblich gedeutet als Grenzfall der relativen Häufigkeit in irgendwelchen repräsentativen Corpora) für die Grundwortstellung erbracht; wir betrachten nur die relevanten Regeln:

(4) Varietätengrammatik (probabilistisch, einfachster Fall von Gewichtung)

$\begin{array}{lllrrrrr} & & & \mathrm{V} 1 & \mathrm{~V}_{2} & \mathrm{~V}_{3} & \mathrm{~V}_{4} & \mathrm{~V}_{5} \\ \text { 1.S }---> & \mathrm{NP} & \mathrm{V} & 0.3 & 0.3 & 0.3 & 0.3 & 0.3 \\ \text { 2. } \mathrm{S} . \longrightarrow & \mathrm{NP} & \mathrm{V} & 0 & 0.1 & 0.2 & 0.4 & 0.5 \\ \text { 3. } \mathrm{S} . \longrightarrow & \mathrm{NP} & \mathrm{NP} & 0.5 & 0.4 & 0.2 & 0.1 & 0 \\ \text { 4. } \longrightarrow & \mathrm{NP} & \mathrm{V} & 0 & 0 & 0.1 & 0.1 & 0.2 \\ \text { 5.S } \longrightarrow & \mathrm{NP} & \mathrm{NP} & 0.2 & 0.2 & 0.2 & 0.1 & 0\end{array}$

In jeder der fünf Varietäten ist, wie gesagt, die Gesamtwahrscheinlichkeit für den ganzen Regelblock 1, denn eine der fünf möglichen Regeln muss ja gewählt werden. Was sich verschiebt, ist die Art, wie sich die Gesamtwahrscheinlichkeit über den gesamten Regelblock verteilt. Für die erste Regel (die die Verbstellung bei intransitiven Sätzen erzeugt) verändert sich nichts: in allen fünf Varietäten hat ein Drittel aller Sätze dieses Muster. Anders ist es bei den transitiven Verben: die Mittelstellung des Verbs steigt allmählich von $0.1 \mathrm{zu} 0.5$; parallel dazu fällt sie für Endstellung des Verbs. Dabei kann es durchaus sein, dass beide Muster gleichzeitig in einer Varietät belegt sind; in $V_{2}$ und $V_{3}$ sind sie sogar gleichwahrscheinlich, sie machen jeweils $20 \%$ aller Satzmuster aus. Entsprechend ist die gegenläufige Verschiebung bei Sätzen mit drei Argumenten. Freilich ist sie nicht vollkommen parallel: das alte Muster hält sich etwas länger; bis $V_{3}$ ist es dominant, in $V_{4}$ zieht es gleich, um dann in $V_{5}$ zu verschwinden.

Auch dieses Beispiel ist sehr einfach; es sollte aber deutlich sein, dass sich durch eine solche probabilistische Gewichtung von Regeln gleitende Übergänge mit beliebiger Genauigkeit abbilden lassen. Gemeinsamkeiten und Unterschiede zwischen Varietäten werden buchstäblich messbar, ohne dass etwas an der formalen Definition der Grammatiken selbst 
geändert werden müsste.

\section{B. Wahl der strukturellen Analyse}

Zugleich zeigt das Beispiel deutlich eine Schwäche dieses Analyse. In die Werte gehen nämlich zwei Faktoren der Variation ein. Das ist zum einen das relative Verhältnis der einzelnen Satzmuster zueinander (einstellige, zweistellige, dreistellige Verben), das andere ist die allmähliche Verschiebung in der Verbstellung bei einem bestimmten Muster, etwa bei den zweistelligen Verben. Beides ist interessant, aber man möchte es trennen können. Die strukturelle Entwicklung, die man erfassen möchte, ist die der Übergang von Verb-End zu VerbZweit. Dies ist jedoch nicht so sehr ein Problem der Varietätengrammatik mit probabilistischer Gewichtung der Regelanwendung, sondern eine Frage der linguistisch sinnvollen "Bezugsgrammatik", also der umfassenden Grammatik, die alle gewichteten Regeln enthält. Im Beipiel ist dies eine simple Phrasenstrukturgrammatik, und (5) stellt auszugsweise eine Gewichtung dieser Bezugsgrammatik dar. Stattdessen kann man sich vorstellen, dass die Phrasenstrukturregeln nur eine einzige Verbstellung vorsehen (sagen wir Anfangsstellung), und die anderen Positionen werden durch eine Transformationsregel erzeugt. Solche Transforma-tionsregeln sind in der Geschichte der generativen Grammatik in vielen Formen vorgeschlagen worden, beispielsweise als Einzelregel, die sich aus "structural index" und "structural change" zusammensetzt (Chomsky 1965), als allgemeines, durch bestimmte Bedingungen beschränktes Prinzip wie "move alpha", wobei alpha eine maximale Projektion ist (Chomsky 1981), oder neuerdings als "move F", wobei F lediglich einen Teil der lexikalischer Merkmale darstellt (Chomsky 1995). Für unsere Diskussion spielt dies keine Rolle; nehmen wir an, $\mathrm{T}_{1}$ sei eine Transformationsregel mit dem Inhalt "Schiebe $\mathrm{V}$ in die Position nach der ersten NP", und $\mathrm{T}_{2}$ sei eine Regel des Inhalts "Schiebe $\mathrm{V}$ in die letzte Position". Dann könnte eine etwas realistischere Varietätengrammatik etwa folgendermaßen aussehen:

(6) Varietätengrammatik mit Basis und Transformationsregeln (probabilistisch)

\begin{tabular}{|c|c|c|c|c|c|c|c|c|c|}
\hline & & & & & V1 & $\mathrm{V} 2$ & V3 & V4 & V5 \\
\hline 1. $\mathrm{S} \longrightarrow$ & $\mathrm{V}$ & NP & & & 0.3 & 0.3 & 0.3 & 0.3 & 0.3 \\
\hline 2. $\mathrm{S} \longrightarrow$ & $\mathrm{V}$ & NP & NP & & 0.5 & 0.5 & 0.4 & 0.5 & 0.5 \\
\hline 3. S $\longrightarrow$ & $\mathrm{V}$ & NP & NP & NP & 0.2 & 0.2 & 0.3 & 0.2 & 0.2 \\
\hline 1 "Schiebe & & y & si & & 0.3 & 0.4 & 0.5 & 0.8 & 1.0 \\
\hline $\mathrm{T}_{2}$ "Schiebe & in & letzt & sit & & .7 & 0.6 & 0.5 & 0.2 & \\
\hline $\mathrm{T}_{3}$ "Lasse $\mathrm{V}$ & a sit & & & & 0 & 0 & 0 & 0 & \\
\hline
\end{tabular}

Hier hat die Bezugsgrammatik zwei Komponenten. Die erste ist eine kontextfreie Basis, in der das Verb immer in Anfangsstellung steht; die zweite besteht aus drei komplementäre Trans-formationsregeln, die wiederum einen Regelblock bilden. Die Basisregeln beschreiben, wie wahrscheinlich ein Satzmuster mit einer bestimmten Argumentstruktur ist; diese Verteilung ist mehr oder minder konstant in allen Varietäten. Von den drei Transformationsregeln läuft die letzte leer (weil hier angenommen wird, dass das Verb nie in Anfangsstellung stehen bleibt). Die beiden andern zeigen eine allmähliche Entwicklung von 
Endstellung zu Zweitstellung. Der aufmerksame Leser wird bemerkt haben, dass wir hier das Satzmuster "NP V" als "Zweitstellung" gerechnet hat; ebensogut hätte man es aber als "Endstellung" ansehen können, weil eben die Zweitstellung gleichzeit die Endstellung ist; dann würden sich die Werte entsprechend verschieben. Dies sieht zunächst einmal wie eine Unzulänglichkeit des Modells aus. In Wirklichkeit aber ist es ein rein strukturelles Problem: was gilt als "Zweitstellung", was als "Endstellung", wenn der Unterschied an der Oberfläche nicht auszumachen ist? Sobald sich dafür Kriterien finden lassen (etwa indem man die Position anderer Elemente, beispielsweise von Adverbien, mit heranzieht), kann man es sich auch in den probabilistischen Gewichtungen abbilden.

\section{Abhängigkeit von Regeln}

In vielen Fällen ist die Anwendung einer Regel davon abhängig, ob zuvor eine bestimmte andere Regel angewandt worden ist. Im obigen Beispiel haben wir einfach von der Verbstellung im "Satz" geredet, unabhängig davon, ob es sich um einen Haupt- oder einen Nebensatz handelt. Nun mögen die Verhältnisse in diesen beiden Fällen ja ganz anders sein; das Neuhochdeutsche ist ja ein schlagendes Beispiel. Dem kann man dadurch Rechnung tragen, dass man nicht von einer einheitlichen Kategorie "Satz" redet, sondern eigene Regeln für "Hauptsatz" und "Nebensatz" ansetzt. Das ist aber nicht sehr ökonomisch; irgendwie möchte man dem Umstand Rechnung tragen, dass es sich in beiden Fällen, bei allen Unterschieden im einzelnen, doch um Sätze handelt. Eleganter ist es daher, zwischen jenen Fällen zu unterscheiden, in denen $S$ "oberstes Symbol" ist, und jenen, in denen $S$ "eingebettet" ist, also etwa als Teil einer NP oder einer Adverbialphrase expandiert wird. Je nachdem können dann die Anwendungs-wahrscheinlichkeiten ganz unterschiedlich sein. Dies läßt sich durch eine einfache probabilistische Bewertung, wie sie oben skizziert wurde, nicht mehr erfassen. Stattdessen muss man mit bedingten Wahrscheinlichkeiten arbeiten. Dafür gibt es unterschiedlich anspruchsvolle Verfahren. Die allgemeinste Lösung besteht darin, jeder Regel der Grammatik einen Namen zuzuordnen, etwa $r_{1}, r_{2}, r_{3}, \ldots, r_{n}$. Die konkrete Ableitung eines Satzes lässt sich dann durch ein "Kontrollwort" darstellen, d.h. eine Folge von Regelnamen, beispielsweise $r_{1} r_{3} r_{4} r_{4} r_{7}$, wobei $r_{1}$ usw. die Regeln sind, die bei der Bildung des Satzes angewandt wurden. Was man dann bewertet, ist die Wahrscheinlichkeit, mit der in einer solchen Ableitung eine bestimmte Regel auf eine andere folgt. Wie dies im einzelnen erfolgt, soll hier nicht im einzelnen dargestellt werden (siehe hierzu Klein 1988, Abschnitt xxx). Es sollte aber deutlich sein, dass sich auf diese Weise Abhängigkeiten beliebiger Art und Komplexität darstellen lassen.

\subsection{Eine Zwischensumme}

Ziel der Varietätengrammatik ist es, von präzise definierten Grammatiken für eine einzelne Varietät zu ganzen Systemen von Grammatiken überzugehen, mit denen sich Ähnlichkeiten und Verschiedenheiten einzelner Varietäten abbilden lassen. Die Varietätengrammatik leistet dies in sehr einfacher Weise, ohne die formale Genauigkeit in irgendeinem Punkt zu opfern. Sie hat ihre Grenzen dort, wo die zugrundeliegenden formalen Grammatiken immanente Unzulänglichkeiten haben. So mag man sich streiten, ob eine Phrasenstrukturgrammatik, sp präzise und elegant sie auch sein mag, wirklich die relevanten Gesetzlichkeiten einer Varietät abbildet. Die meisten Linguisten würden dies heute nicht mehr annehmen, obwohl die Argumente in der einen wie in der andern Hinsicht dürftig sind. Aber wie oben zumindest angedeutet wurde, lässt sich das Vorgehen ohne weiteres auch auf andere Grammatikmodelle 
übertragen, sofern diese genau definiert sind. Eine Varietätengrammatik kann, was die Adäquatheit der strukturellen Analyse angeht, nicht besser sein als die Art von Grammatik, auf der sie beruht. Aber sie leistet alles, was diese leistet, und einiges mehr: sie erlaubt es, die gesamte Heterogenität der Sprache - der "Wundt-Sprache" wie der "Paul-Sprache" - präzise abzubilden und Gemeinsamkeiten wie Verschiedenheiten der einzelnen Varietäten messbar zu machen. Sie ist mithin unabhängig von der Art und Weise, wie man den Gegenstand der Sprachwissenschaft konzeptualisiert, und dies ohne besonders starke Idealisierungen, die zwar oft erforderlich, aber empirisch allemal problematisch sind.

\section{Warum hat sie sich nicht durchgesetzt?}

Nach all dem, was im vorigen Abschnitt gesagt wurde, ist die Varietätengrammatik eigentlich ein ideales Instrument für die Analyse der sprachlichen Variabilität im Bereich der Grammatik. Sie ist auch in einer Reihe von Fällen angewandt worden (Tropf 1983 in der Phonologie, Senft 1982 und Carroll 1984 in der Syntax). Aber im Grunde ist sie tot, wie so vieles andere in der Linguistik der letzten dreißig Jahre. Warum?

Wir sind geneigt, den Erfolg einer wissenschaftlichen Entdeckung, einer bestimmten Analyse, einer ganzen Theorie mit ihren inhärenten Verdiensten in Zusammenhang zu bringen: ist sie originell, ist sie wahr, erklärt sie vieles, was sich anderweitig nicht erklären lässt, hat sie zumindest deskriptive Meriten.. Ich glaube, das ist ein sehr naives Bild vom Werdegang einer Wissenschaft. Er wird sicher von der Suche nach der Wahrheit und nach tiefern Einsichten bestimmt, aber nicht minder von den Mäandern der Mode und den Gesetzen des Marktes, auf dem die Forscher ihre Produkte feilbieten. Mir scheint, für den Umstand, dass sich weder in der theoretischen Linguistik noch in der Soziolinguistik - wie denn in der Varietätenforschung allgemein - kaum jemand der Varietätengrammatik bedient, kann man drei Gründe anführen: inhärente Schwächen, den Zeitgeist und das Marketing.

\subsection{Inhärente Schwächen}

Im vorausgehenden Abschnitt wurden bereits einige Probleme der Varietätengrammatik angeführt, die sich aber, wie dort zumindest angedeutet wurde, prinzipiell lösen lassen. Es bleiben drei weitere. Zum einen hat die Varietätengrammatik in sich keinen Erklärungswert. Sie beschreibt einfach die einzelnen Varietäten, ihre innere Struktur und ihr Verhältnis zueinander. Dies erklärt aber beispielsweise nicht, wieso - um bei dem obigen Beispiel zu bleiben - das Verb allmählich vom Satzende in die zweite Position rückt. Ebensowenig kann sie erklären, weshalb man in einem Soziolekt eine bestimmte Struktur favorisiert, in einem anderen hingegen eine andere. Dies kann in sich das Ergebnis einer historischen Entwicklung sein - ein Beispiel für eine Paulsche Erklärung; es kann aber auch andere Faktoren reflektieren, wie dies etwa für die unterschiedliche Stellung des Verbs in weil-Sätzen oft angenommen wird. Die probabilistische Beschreibung läßt dies einfach offen. Allerdings schließt sie auch keinerlei Erklärung aus, ebensowenig wie eine präzise Beschreibung einer homogenen Sprache weitere Betrachtungen darüber ausschließt, welche historischen oder psychologischen Gründe dazu geführt haben, dass sie so ist, wie sie ist.

Zum zweiten erhebt die Varietätengrammatik nicht den Anspruch der "psychologische Realität". Die Regeln decken einfach die beobachtbaren Fakten ab; es mag ganz andere Regelformulierungen geben, die dieselben Fakten abdecken. Dann würde man jene wählen, die am einfachsten sind oder die die Unterschiede zwischen den einzelnen Varietäten am 
deutlichsten sichtbar machen; wir haben dies weiter oben in der Umformulierung von einer reinen Phrasenstrukturgrammatik zu einer "Basis plus Transformationsregeln" illustriert. Eine ganz andere Frage ist, ob ein Sprecher, der beide Varietäten in seinem Kopf gespeichert hat, dies dort genau so repräsentiert. Wenn man es für das Ziel der Linguistik hält, das Wissen des Sprechers abzubilden, so wie er es nun einmal im Kopf hat, dann ist dies ein Manko. Allerdings gilt auch hier, dass die Varietätengrammatik mit dieser Ambition nicht unverträglich ist; sie ist in der Tat, was diesen Punkt angeht, sogar realistischer als eine normale, auf der Homogenitätsannahmen beruhende Analyse - denn das Wissen des Sprechers ist normalerweise nicht homogen. Und schließlich möchte man auch, gleichwelcher linguistischen Sekte man anhangt, oft auch Varietäten miteinander vergleichen, deren Kenntnis nicht zur Kompetenz irgendeines lebenden oder toten Sprechers gehört beispielsweise die Entwicklung der deutschen Wortstellung über die Jahrhunderte hinweg.

Zum dritten wird sie, wenn die empirischen Gegebenheiten komplex sind, auch ihre Darstellung komplex und umständlich. Dies halte ich für das ernsthafteste Problem. Wir haben in dem obigen Beispiel die realen Verhältnisse sehr vereinfacht; Verbflexion und Kasusmarkierung sind nicht berücksichtigt, andere Konstituenten können intervenieren, Faktoren wie die Topik-Fokus-Struktur spielen eine Rolle, usw. usw. Dies mit den Mitteln einer formalen Grammatik präzise zu erfassen, ist nicht einfach. Entsprechend schwierig wird eine Beschreibung über verschiedene Varietäten hinweg, und erst recht komplex wird es, wenn man beispielsweise die Abhängigkeit von Regeln berücksichtigen will. Aber dies ist ganz einfach der Preis, den man für Genauigkeit zahlt. Es ist allemal einfacher und oft auch vorteilhafter, die Dinge ein wenig im Ungefähren zu belassen.

\subsection{Der Zeitgeist}

Wenn man die Entwicklung der Linguistik in den letzten drei Jahrzehnten insgesamt betrachtet, so fällt auf, dass das Interesse an expliziten formalen Beschreibungen in der Syntax weitestgehend geschwunden ist. Die generative Grammatik beispielsweise hatte zu Beginn der Siebzigerjahre eine recht präzise Grundlage in der Theorie der formalen Sprachen, die wesentlichen Eigenschaften von kontextfreien Grammatiken, Kategorialgrammatiken, Transformationsgrammatiken waren bekannt, und es wurde versucht, die strukturellen Eigenschaften einzelner (als homogen angenommener) Sprachen mit diesen Methoden zu fassen. Dies ist nicht mehr der Fall; zwar tauchen immer noch Strukturbäume in den Aufsätzen auf, aber es sind eher mehr oder minder einleuchtende Illustrationen. Über die Eigenschaften der zugrundeliegenden formalen Systeme ist wenig bekannt, und nur wenige interessieren sich überhaupt dafür. Diese Entwicklung hat eine Reihe von Gründen, die wir hier nicht verfolgen können. Formale Methoden haben sich eigentlich nur in der Semantik, vor allem aber in der Computerlinguistik, wo präzise Formulierungen unabdinglich sind, gehalten. Das nachlassende Interesse an der Varietätengrammatik liegt daher voll im Zug der Zeit: niemand interessiert sich dafür, die strukturellen Eigenschaften genau auszubuchstabieren. Das mag man für gut oder schlecht halten - aber es ist keine Sonderentwicklung in der Varietätenforschung. Wenn es aber eine Besonderheit gibt, dann liegt er sicher in der emotialen Verhätlnis der sprachwissen-schaftlich Tätigen zu Wahrscheinlichkeiten. Der Linguist ist, hierin ein klassischer Geistes-wissenschaftler, quantitativen Methoden gegenüber durchweg mißtrauisch; er versteht sie in der Regel auch nicht. Dies gilt ganz besonders für probabilistische Methoden - bis hin $\mathrm{zu}$ absurden Argumenten wie, dass die Sprecher nicht zählen und rechnen (als ob ein Würfel, der mit der 
Wahrscheinlichkeit von 1/6 auf die "zwei" fällt, darüber Buch führen würde, wie oft er schon auf welche Zahl gefallen ist). Auch hier hat es vor zwei, drei Jahrzehnten viele Bemühungen gegeben, das Spektrum der linguistischen Methoden dem Vorgehen anderer empirischer Wissen-schaften anzunähern (die anderen Beiträge in diesem Band enthalten eine Menge einschlägiger Referenzen). Sie sind erfreulicherweise nicht ganz versandet, stehen aber nicht eben im Rampenlicht der sprachwissenschaftlichen Forschung.

\subsection{Marketing}

Wenn jemand eine neue Zahnpasta erfindet oder ein neues Betriebssystem für Heimrechner, so besagt der Umstand, dass es die Zähne sauberer putzt oder den Rechner besser verwaltet, noch wenig darüber, ob es die Aufmerksamkeit der vielen findet. Man muss es "am Markt durch-setzen", und der Erfolg dort folgt nicht den Kriterien der natürlichen, sondern jenen der künstlichen Selektion. Das ist in den reinen Wissenschaften anders und auch wieder nicht. Galilei und Newton haben Lug und Trug nicht verschmäht, um ihre Ansichten auf dem Markt der Ideen durchzusetzen. Aber sie hatten, wie sich nachher gezeigt hat, in wesentlichen Punkten auch recht, ihre Ansichten haben sich bestätigt und bewähr, der Zweck hat die Mittel in Grenzen geheiligt. Die Theorie der natürlichen Selektion durchzusetzen, war nicht zuletzt ein Erfolg eines kleines Netzwerks von "old boys", die Darwin ihre Unterstützung gegeben haben; aber diese Theorie ist auch gut, wenngleich vielleicht etwas inhaltsleer und schlecht zu falsifizieren. Heute, zu Ende des 20. Jahrhunderts, ist der Wissenschaftsbetrieb jedoch in unvergleichlich höherem Maße von Marktgesetzen bestimmt. Damit will ich keineswegs sagen, dass die amtlichen Kriterien wie Wahrheit, Klarheit, Innovatität in der Wissenschaft ganz irrelevant geworden wären. Aber sie haben relativ an Gewicht verloren gegenüber den Anforderungen des Marktes. Dies gilt für alle Wissenschaften, die "harten" wie die "weichen", es gilt auch für das relativ kleine Fach der Sprachwissenschaft. Wer kennt wen, wer veröffentlicht wo, wer zitiert wen, wer promoviert bei wem, wer erfasst den Zeitgeist am besten? Wer lanciert seine Vorstellungen am geschicktesten ins Sommerloch der Theoriendiskussion? Gut anderthalb Jahrzehnte hat in der Linguistik die Idee von der "Modularität des Geistes" eine eminente Rolle gespielt; nun ist sie fast völlig aus der Diskussion verschwunden, ohne erkennbaren Grund, so wie sie ohne erkennbaren Grund Furore auf dem Forum gemacht hat.

\section{Was können wir daraus lernen?}

Nur ein sehr einfältiger Mensch kann glauben, die Interessen und Methoden, die das Bild einer Wissenschaft $\mathrm{zu}$ einer gewissen Zeit bestimmen, verdankten dies vorwiegend der internen Entwicklung der Wissenschaft selbst. Den Erfolg oder Misserfolg einer bestimmten Auffassung, einer bestimmten Theorie allein aus ihrer größeren Adäquatheit oder größeren Exaktheit zu erklären, gleicht der Teleologie eines Fussballreporters, für den der Gewinner immer zu Recht mehr Tore geschossen hat. In Wirklichkeit ist vieles einfach Zufall, Wandel der Mode, gutes "product placement". Dies gilt in meinen Augen auch für die Varietätengrammatik, wie für so gut wie alle anderen Versuche, die sprachliche Variation mit präzisen Methoden zu erfassen. Kann man daraus etwas lernen? Ich habe nicht die geringste Ahnung. 
Literaturhinweise

Ammon, Ulrich / Dittmar, Norbert / Mattheier, Klaus (edd.) (1987-1988): Sociolinguistics. Berlin/New York: de Gruyter.

Dittmar, Norbert (1997): Grundlagen der Soziolinguistik - ein Arbeitsbuch mit Aufgaben. Tübingen: Niemeyer.

Bierwisch, Manfred (1976): Social differentiation of language structure. In Asa Kasher, ed., Language in Focus, Amsterdam: Benjamins, S. 407-456.

Carroll, Mary (1984): xxx

Chomsky, Noam (1965): Aspects of the Theory of Syntax. Cambridge, Mass.: MIT Press.

Chomsky, Noam (1981): Lectures on Goverment and Binding. Amsterdam: Foris.

Chomsky, Noam (1986): Knowledge of Language. New York: Prager.

Chomsky, Noam (1995): The Minimalist Program. Cambridge, Mass.: MIT Press.

Habel, Christopher (1979): Aspekte bewertender Grammatiken. Berlin: Einhorn.

Klein, Wolfgang (1974): Variation in der Sprache. Kronberg: Scriptor.

Klein, Wolfgang (1988): Varietätengrammatik. In: Ammon et al. (1987-1988), S. 997-1006.

Labov, William (1972a): Language in the inner city. Philadelphia: University of Pennsylvania Press.

Labov, William (1972b): Black English Vernacular. Philadelphia: University of Pennsylvania Press.

Paul, Hermann (1880): Principien der Sprachgeschichte. Jena: Niemeyer (2. Aufl. ebd. 1886, 4. $\quad$ Auflage ebd. 1909).

Senft, Gunter (1982): Sprachliche Varietät und Variation im Sprachverhalten Kaiserslauterner Metallarbeiter. Frankfurt: Lang.

Tropf, Herbert (1983): Variation in der Phonologie des ungesteuerten Zweitspracherwerbs. Heidelberger Phil. Diss.

Wundt, Wilhelm (1900): Die Sprache. Leipzig: Engelmann. 